\begin{tabular}{|c|c|}
\hline 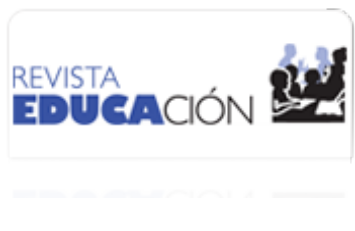 & $\begin{array}{l}\text { Revista Educación } \\
\text { ISSN: 0379-7082 } \\
\text { ISSN: } 2215-2644 \\
\text { revedu@gmail.com } \\
\text { Universidad de Costa Rica } \\
\text { Costa Rica }\end{array}$ \\
\hline
\end{tabular}

\title{
Metodologías activas: una necesidad en la Unidad Educativa Reino de Inglaterra
}

\author{
Cano de la Cruz, Yullio; Aguiar Monar, Jenniffer Cristina; Mendoza Román, Mishell Carolina \\ Metodologías activas: una necesidad en la Unidad Educativa Reino de Inglaterra \\ Revista Educación, vol. 43, núm. 2, 2019 \\ Universidad de Costa Rica, Costa Rica \\ Disponible en: http://www.redalyc.org/articulo.oa?id=44058158037 \\ DOI: https://doi.org/10.15517/revedu.v43i2.29094
}

Esta obra está bajo una Licencia Creative Commons Atribución-NoComercial-SinDerivar 3.0 Internacional. 


\section{Metodologías activas: una necesidad en la Unidad Educativa Reino de Inglaterra}

\section{The Need for Active Methodologies at the Reino de Inglaterra School}

Yullio Cano de la Cruz

Pontificia Universidad Católica del Ecuador, Sede Santo

Domingo, Ecuador

ccy@pucesd.edu.ec

(iD http://orcid.org/0000-0001-6315-1488

\section{Jenniffer Cristina Aguiar Monar \\ Unidad Educativa Particular Federico González Suárez,}

Ecuador

jcaguiarm@pucesd.edu.ec

iD http://orcid.org/0000-0002-5041-4573

\author{
Mishell Carolina Mendoza Román \\ Unidad Educativa Particular Raúl González Astudillo, \\ Ecuador \\ mcmendozr@pucesd.edu.ec \\ http://orcid.org/0000-0002-4666-7949
}

DOI: https://doi.org/10.15517/revedu.v43i2.29094 Redalyc: http://www.redalyc.org/articulo.oa?id=44058158037
Recepción: 29 Mayo 2017

Aprobación: 19 Junio 2019

\section{Resumen:}

La necesidad de ambientes de aprendizaje sustentados en metodologías activas que conduzcan al logro de los estándares de la calidad educativa establecidos por el Ministerio de Educación en Ecuador, generó la siguiente investigación la cual tuvo como objetivo diseñar un módulo de capacitación en el uso de metodologías activas, mediante la aplicación de talleres para la formación del cuerpo docente de Educación General Básica de la Unidad Educativa Reino de Inglaterra. La metodología consistió en un diseño cuasiexperimental de un solo grupo al cual se le aplicó un pretest y un postest, bajo un enfoque cuantitativo. La muestra fue no probabilística intencional consistente en 14 docentes de la unidad educativa, a quienes se les aplicó un pretest y un postest elaborados por las personas autoras y validado por criterio de experticia. El pretest arrojó como resultados un insuficiente conocimiento por parte del grupo docente de la muestra acerca de las metodologías activas, información que condujo al diseño del módulo de capacitación y aplicación de los talleres. Posteriormente se cerró la investigación con la aplicación de un postest que reveló una media aritmética alta, la cual demostró la efectividad del módulo de capacitación en el conocimiento de las metodologías activas.

Palabras Clave: Metodología, Enseñanza, Aprendizaje, Docente, Capacitación.

\section{Abstract:}

The need for more learning environments focused on active methodologies, part ofan effort by the Ministry of Education in Ecuador to improve quality standards ofthe country's educational system, served as the basis for the following study. The study endpoint was to design a training module that would effectively teach the use of active methodologies through workshops for training Primary School Teachers from the Reino de Inglaterra Elementary School. The methodology consisted of a quasiexperimental design with a quantitative focus for a single group that was asked to take a pretest and a posttest. The sample was intentionally non probabilistic, consisting of 14 teachers at the school who were asked to take a pretest and a posttest. The pretest did not provide significant results regarding the teacher sample group knowledge about active methodologies. This information gap was the basis of a workshops training module. We concluded the study with the posttest which revealed a high arithmetic mean validated the effectiveness of the training module to increase knowledge about active methodologies.

KEYWORDS: Methodology, Teaching, Learning, Teacher Training. 


\section{INTRODUCCIÓN}

El desarrollo de una educación de calidad es una de las prioridades de los sistemas educativos a nivel mundial, contexto al cual la República del Ecuador no está ajena. Para su logro se establece dentro de los objetivos del Plan Nacional del Buen Vivir (Secretaría Nacional de Planificación de la República del Ecuador, 2013) (Sumak Kawsai en quichua) el logro de la Calidad Educativa, basada en la formación individual y en la equidad.

Sin embargo, los resultados obtenidos en el Tercer Estudio Regional Comparativo y Explicativo de la Calidad de la Educación (TERCE) desarrollado en julio del 2015 por el Laboratorio Latinoamericano de Evaluación de la Calidad de la Educación (LLECE), evidencian que en materias como Lectura y Escritura los resultados están por debajo de la media regional. En otras materias como Matemáticas y Ciencias Naturales solo se alcanzan la media regional y en ningún caso se supera. (Flotts, Manzi, Jiménez, Abarzúa, Cayuman y García, 2016)

Estos resultados son producto de una problemática dada en Ecuador relacionada con la insuficiente capacitación a docentes, sobre todo a quienes no tienen una titulación de tercer nivel en educación y utilizan métodos tradicionales que generan un aprendizaje reproductivo y con poca significatividad, impidiendo el cumplimiento de los estándares establecidos por el Ministerio de Educación de Ecuador (MINEDUC). El diario El Comercio, en su tirada del 30 de julio de 2016 resalta que el resultado de la evaluación al profesorado de planteles fiscales evidencia fortalezas y debilidades de una planta que no logra ser del siglo XXI. (Castillo y Puente, 2016)

Ante esta situación, el MINEDUC establece lineamientos para la formación continua y capacitación a docentes. El artículo 10 de la Ley Orgánica de Educación Intercultural (2011), en su literal A, establece que el cuerpo docente del sector público tiene como derecho: "Acceder gratuitamente a procesos de desarrollo profesional, capacitación, actualización, formación continua, mejoramiento pedagógico y académico en todos los niveles y modalidades, según las necesidades del Sistema Nacional de Educación” (p. 14).

No obstante, aun cuando existe esta apertura a la capacitación y actualización docente, datos revelados por el diario El Comercio indican que, de los 48.835 docentes de la Sierra y la Amazonía que participaron en los procesos de capacitación, el 5,5\% reprobó, estos datos indican que la problemática de la capacitación docente está latente en el contexto educativo ecuatoriano. Por otro lado, es limitado el número de cupos respecto a la demanda, lo cual requiere que las instituciones educativas generen sus propios espacios de capacitación en función de sus necesidades.

Si a esto se le suma que los cursos de capacitación ofertados por el MINEDUC en el 2016 y 2017 estuvieron relacionados con propuestas curriculares abiertas y flexibles para atender la diversidad en las aulas; el currículo nacional y la planificación en su segundo y tercer nivel y la evaluación en el aula, se evidencia una clara necesidad de que las instituciones educativas ejecuten capacitaciones sobre metodologías activas que le permitan lograr un aprendizaje significativo y el cumplimiento de los estándares de calidad.

Por su parte, Díaz (2006) en su investigación: Formación docente, práctica pedagógica y saber pedagógico, plantea un cuestionamiento directo: ¿Cuál es el rol que están tomando los y las docentes en la actualidad? ¿Tienen estos las competencias y preparación necesaria para llevar a efecto el acto de la enseñanza?, precisando que la formación docente y la actualización, son focos de vital importancia para alcanzar los objetivos educativos que se persiga.

Con mención a las metodologías activas, De León (2013) denota las deficiencias en el rendimiento académico del estudiantado por la falta de su utilización en la Educación General Básica. Además, alude que su empleo fomenta el desarrollo de un aprendizaje de calidad.

Del mismo modo, Barrado (citado por Campillay y Meléndez, 2015), nombra que es indispensable aplicar un aprendizaje activo porque mejora el nivel de atención en el estudiantado dejando que ellos sean autónomos 
y responsables en la adquisición de los nuevos conocimientos, fortaleciendo su pensamiento analítico y crítico.

Estos antecedentes apuntan hacia las potencialidades que ofrecen las metodologías activas; sin embargo, la problemática de la capacitación docente al respecto necesaria para el logro de una educación de calidad condujo a formular como objetivo de la presente investigación: diseñar un módulo de capacitación en el uso de metodologías activas, mediante la aplicación de talleres para la formación de docentes de Educación General Básica de la Unidad Educativa Reino de Inglaterra.

\section{Marco Teórico}

En la Didáctica, el método es el conjunto de procedimientos que se emplea en el aula para alcanzar los objetivos propuestos para la clase y desarrollar las destrezas con criterios de desempeño. Según Escudero (2013), se ha de seguir un buen método didáctico porque el fin de la tarea es el desenvolvimiento armónico de las habilidades del estudiantado y la transmisión de un conjunto de conocimientos lógicamente encadenados. Sobre lo anterior, se considera al método como el camino para lograr una consecuencia y alcanzar los objetivos.

En la actualidad se maneja una amplia clasificación de los métodos donde las metodologías activas ocupan un lugar esencial. A criterio de Labrador (2008) las metodologías activas son aquellos métodos, técnicas y estrategias los cuales utiliza el personal docente para convertir el proceso de enseñanza en actividades que fomenten la participación activa del estudiantado y conduzcan a un aprendizaje significativo.

Existen numerosas metodologías que responden al criterio de activas; no obstante, para la presente investigación se establecen como metodologías activas la simulación, el aprendizaje por experiencia, el aprendizaje basado en problemas y el aprendizaje cooperativo. Profundizando en cada uno de ellos permite plantearse al aprendizaje por simulación como un método que favorece la adquisición de conocimientos especializados, al tiempo que permite interactuar, comunicar, liderar o trabajar en equipo en situaciones ficticias en contextos reales (García, 2012).

Por su parte, el aprendizaje por experiencia, como su nombre lo indica, tiene su mira fija en la relevancia del papel que juega la experiencia o vivencia en el proceso de enseñanza - aprendizaje. Desde este punto de vista, el aprendizaje es un proceso de reflexión y de dar sentido a las experiencias (Romero, 2010).

En tanto, en el aprendizaje basado en problemas se le presenta una problemática al estudiantado, a la cual deben dar solución. Desde el punto de vista procedimental se basa en la investigación, interpretación, argumentación y propuesta de solución a un problema que alienta a participar en escenarios relevantes que facilitan la aplicación de la teoría aprendida. (Learreta, Montiel,González y Asensio, 2009).

Por último, el aprendizaje cooperativo es el proceso por el cual la membresía de un equipo alcanza objetivos compartidos y básicamente se refiere a la actividad de pequeños equipos que desarrollan una actividad de aprendizaje y reciben evaluación de los resultados conseguidos (Morera, Climent, Iborra citado por Learreta et àl., 2009).

Los métodos son el elemento más dinámico del proceso de enseñanza-aprendizaje, sin embargo, por sí solos no permiten el cumplimiento de los objetivos, por lo que es necesario complementarlos con las técnicas de enseñanza. Estas se consideran como las acciones que desarrolla cada docente que tienden a provocar determinadas modificaciones en los educandos en función del logro de los objetivos propuestos. Esencialmente, son el conjunto de procedimientos, tácticas o recursos concretos, previamente planificados para ser utilizados con la finalidad de alcanzar los objetivos de aprendizaje en el estudiantado. (Vargas, 2009)

Por otra parte, las técnicas de aprendizaje son procedimientos que se usan de un modo intencional y premeditado a una tarea y que no pueden reducirse a rutinas automatizadas, es decir, son más que simples series de habilidades; las técnicas tienen un carácter intencional; implican, por tanto, un plan de acción (Herrera y Lorenzo, 2009). 
Los referentes teóricos anteriores demuestran la importancia de los métodos y las técnicas en el proceso de enseñanza-aprendizaje, por lo que es necesario desarrollar capacitaciones encaminadas a su empleo adecuado, a un cambio de actitud ante la situación intercultural y el aumento de la heterogeneidad del estudiantado. Para Grados (citado por Aguilar, 2010), señala que la capacitación es la acción interesada en desplegar los conocimientos, habilidades y actitudes del trabajador con el fin de prepararlo para que se desempeñe correctamente en un puesto específico de trabajo.

El claustro docente necesita de actualización constante y permanente con el fin de mejorar la calidad educativa. Es por ello que se presenta en un determinado espacio el desempeño para analizar su metodología y capacidad de enseñanza que permita impartir una correcta formación del aprendizaje.

La educación va mejorando con el paso del tiempo, por ende, es fundamental la formación docente, pues se capacita al profesorado con nuevas visiones a futuro que enmarca principalmente la formación integral del estudiantado, por medio de innovadoras estrategias y métodos de enseñanza aprendizaje.

\section{Procedimientos metodológicos}

La investigación se desarrolló bajo un enfoque de investigación cuantitativo, siendo esta de tipo explicativo con un diseño cuasiexperimental de un solo grupo con pretest y postest. Se manipuló en grado de presencia la variable independiente talleres de capacitación para observar su efecto en la variable dependiente insuficiente capacitación en metodologías activas. Considerando que en los cuasiexperimentos los sujetos no se asignan al azar a los grupos, ni se emparejan, sino que son grupos intactos conformados antes del experimento (Hernández, Fernández y Batista, 2014) la muestra utilizada fue no probabilística intencional, consistente en los 14 docentes de ambos sexos de la Unidad Educativa Reino de Inglaterra.

Como instrumentos de recogida de datos se aplicó a la muestra un pretest y un postest. El pretest consistió en un cuestionario de 10 ítems en el cual, los cuatro primeros respondieron al indicador conocimientos sobre metodologías activas. En su elaboración se utilizó las preguntas de selección múltiple, donde a partir de un caso presentado se debía identificar la metodología utilizada. Para justificar la necesidad de la manipulación en grado de presencia de la variable independiente talleres de capacitación sobre metodologías activas el resto de los seis ítems respondieron equitativamente a los indicadores: participación en capacitaciones sobre metodologías activas y necesidad de talleres de capacitación sobre metodologías activas. En estos ítems se utilizó el escalamiento de Likert y el diferencial semántico de Osgood. Por su parte, el postest consistió en otro cuestionario con 10 ítems sobre el indicador conocimientos sobre metodologías activas.

Ambos instrumentos fueron elaborados por los autores y aun cuando tuvieron un carácter ad hoc, considerando que con el estudio no se persiguió efectuar generalizaciones probabilísticas por el reducido número de la muestra, se les determinó su validez. Para ello primeramente se determinó la validez de contenido correlacionando los indicadores con los ítems, y luego la validez estadística mediante la fórmula Razón de Validez del Contenido sobre el criterio de los siete jueces seleccionados para la validación (Lawshe, 1975) cuyo resultado se interpretó mediante el índice de Lynn (1986), lo cual permitió definir los ítems finales de cada instrumento. Para el análisis de los datos se empleó la estadística descriptiva basada en la media aritmética y el cálculo porcentual.

Como procedimiento, primeramente, se ingresó un oficio solicitando la autorización de los directivos de la Unidad Educativa Reino de Inglaterra para la aplicación de la investigación, una vez recibida la respuesta afirmativa se procedió a la aplicación de la encuesta y el pretest en una primera fase. Posteriormente a esto se ejecutaron los talleres en una segunda fase, para culminar con la aplicación del postest. 


\section{ANÁLISIS Y DISCUSIÓN DE LOS RESULTADOS}

En la Tabla 1 aparecen los resultados de los ítems 1 al 4, estos se calificaron de la siguiente manera: al dominio de las metodologías activas se le asignó una calificación de tres puntos, al dominio medio se le asignó dos puntos y al poco dominio o ninguno se les asignó una calificación de un punto. La calificación cuantitativa se relacionó con la cualitativa de la siguiente manera: tres puntos equivalen a estar en un nivel Alto, dos puntos a un nivel Medio y un punto a un nivel Bajo en cada ítem. Con las puntuaciones por ítems en cada nivel se determinó la media aritmética de docentes por nivel y el porcentaje que representan de la muestra.

De esta manera, como se observa en la Tabla 1 , los niveles de conocimiento acerca de las metodologías activas en docentes de la muestra presentan medias entre Altas y Bajas, siendo las más elevadas las del nivel Bajo, lo que indica no tener conocimientos suficientes sobre las metodologías activas.

Resulta interesante destacar los resultados obtenidos en el ítem 4 el cual tuvo el mayor número de docentes de la muestra evaluado con una calificación de Alto, dado que la metodología activa más conocida es el Aprendizaje Basado en Problemas (ABP) el cual logran identificar, sin embargo, en cuanto al reconocimiento de su procedimiento de aplicación presentan falencias, lo cual demuestra que, aun cuándo lo conocen, no lo aplican en el aula o lo hacen de manera incorrecta.

TABLA 1

Datos estadísticos de los ítems 1 al 4 según el Indicador Conocimiento sobre Metodologías Activas. (Ejecución Actividades con los Tipos de Aprendizaje por Experiencia, Cooperativo, ABP y Simulación)

\begin{tabular}{|c|c|c|c|c|c|c|c|}
\hline \multirow[t]{2}{*}{ Nivel } & \multirow{2}{*}{$\begin{array}{l}\text { Relación escala valorativa-opción de } \\
\text { respuesta. }\end{array}$} & \multicolumn{4}{|c|}{ Ítems } & \multirow{2}{*}{$\begin{array}{l}\text { Media } \\
\text { aritmética }\end{array}$} & \multirow{2}{*}{$\begin{array}{l}\% \\
\text { respecto } \\
\text { a la } \\
\text { muestra }\end{array}$} \\
\hline & & 1 & 2 & 3 & 4 & & \\
\hline Alto & $\begin{array}{l}\text { Domina a profundidad las } \\
\text { metodologias activas. }\end{array}$ & 6 & 3 & 1 & 7 & 5 & $35,71 \%$ \\
\hline Medio & $\begin{array}{l}\text { Identifica ciertas características de } \\
\text { las metodologias activas. }\end{array}$ & 0 & 0 & 4 & 0 & 1 & $7,14 \%$ \\
\hline Bajo & $\begin{array}{l}\text { No conoce sobre metodologías } \\
\text { activas }\end{array}$ & 8 & 11 & 9 & 7 & 9 & $64,2 \%$ \\
\hline
\end{tabular}

Fuente: Elaboración propia

En cuanto a los indicadores participación en capacitaciones sobre metodologías activas y necesidad de talleres de capacitación sobre metodologías activas, las Tabla 2 y Tabla 3 muestran que las frecuencias más altas están en correspondencia con el grupo docente que no han recibido capacitaciones al respecto (42,86\%) y la necesidad de organizar los talleres de capacitación (100\%)

\section{TABLA 2}

Frecuencia con que han recibido los y las docentes de la muestra capacitaciones sobre metodologías de enseñanza y aprendizaje

\begin{tabular}{lll}
\hline Opciones & Frecuencia & Porcentaje \\
\hline 1 vez al año & 3 & $21,43 \%$ \\
\hline Cada 2 años & 5 & $35,71 \%$ \\
\hline No ha recibido & 6 & $42,86 \%$
\end{tabular}

Fuente: Elaboración propia 
TABLA 3

Necesidad de capacitaciones sobre metodologías activas para los y las docentes de la Unidad Educativa Reino de Inglaterra

\begin{tabular}{lll}
\hline Opciones & Frecuencia & Porcentaje \\
\hline Si & 14 & $100,00 \%$ \\
\hline No & 0 & $0,00 \%$ \\
\hline Total & 14 & $100,00 \%$ \\
\hline
\end{tabular}

Fuente: Elaboración propia

Como segunda etapa del cuasiexperimento se manipuló la variable independiente, partiendo del diseño de un módulo de capacitación consistente en cuatro talleres (Ver Tabla 4 y Tabla 5).

\section{Perfil de egreso del módulo de capacitación:}

\section{Saber:}

Comprende las diferentes metodologías activas que sustentan las planificaciones curriculares

Saber hacer:

Elabora planificaciones curriculares con un enfoque activo.

\section{Saber conocer:}

Construye el conocimiento de la realidad educativa desde un enfoque activo.

\section{Saber ser:}

Actúa críticamente, basándose en la ética pedagógica.

Elementos estructurales de las Competencias:

\section{Problema:}

¿Cómo elaborar planificaciones curriculares activas?

\section{Competencias:}

C1. Adquirir conocimientos sobre metodologías activas para elaborar planificaciones curriculares que le permitan generar un aprendizaje significativo.

\section{Rango de aplicación:}

Nivel micro curricular.

TABLA 4

Estructura del módulo de capacitación. Relación competencias-resultados de aprendizaje-contenidos

\begin{tabular}{|c|c|c|}
\hline Competencia & $\begin{array}{l}\text { Resultados de } \\
\text { aprendizaje }\end{array}$ & Contenidos \\
\hline $\begin{array}{l}\text { C1. Adquirir } \\
\text { conocimientos } \\
\text { sobre } \\
\text { metodologias } \\
\text { activas para } \\
\text { elaborar } \\
\text { planificaciones } \\
\text { Curriculares que le } \\
\text { permitan generar } \\
\text { un aprendizaje } \\
\text { significativo. }\end{array}$ & $\begin{array}{l}\text { Caracteriza las } \\
\text { metodologias activas. } \\
\text { Elabora planificaciones } \\
\text { curriculares sustentadas } \\
\text { en metodologias activas. }\end{array}$ & $\begin{array}{l}\text { 1. Metodologías activas. Definición } \\
\text { y tipología. } 2 \text {. Aprendizaje por } \\
\text { simulación. Definición. Procedimiento. } \\
3 . \quad \text { Aprendizaje cooperativo. } \\
\text { Definición. Procedimiento. } 4 \text {. } \\
\text { Aprendizaje basado en problemas. } \\
\text { Definición. Procedimiento. } 5 \text {. } \\
\text { Aprendizaje por experiencia. Definición. } \\
\text { Procedimiento. }\end{array}$ \\
\hline
\end{tabular}




\section{Metodología:}

La metodología de enseñanza se desarrollará mediante cuatro talleres (Ver Tabla 5).

\section{Evaluación:}

La evaluación será mediante la aplicación del postest.

Descripción de la realización de los talleres:

TABLA 5

Estructura de los talleres.

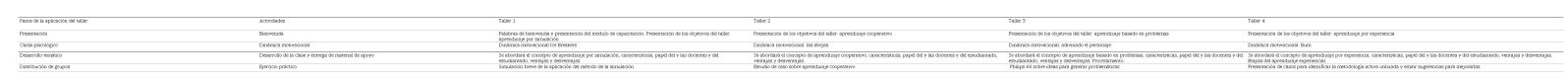

Fuente: Elaboración propia

Como última fase del diseño cuasiexperimental seguido se procedió a la aplicación del postest. En la Tabla 6 se muestran los datos estadísticos de su aplicación. El procesamiento se desarrolló de similar manera que el pretest en cuando a la calificación y la asignación de equivalencias cualitativas de alto, medio y bajo. Cobra significancia observar como la media aritmética indica una prevalencia de un nivel alto, en función de esto, se logró que el 92, 85\% de la muestra adquiriera conocimientos sobre las metodologías activas.

TABLA 6

Resultados del postest. Indicador conocimiento sobre metodologías activas

\begin{tabular}{|c|c|c|c|c|c|c|c|c|c|c|c|c|c|c|c|c|}
\hline \multirow{2}{*}{ Nivel } & \multirow{2}{*}{$\begin{array}{l}\text { Relación escala } \\
\text { valorativa-opción } \\
\text { de respuesta }\end{array}$} & \multicolumn{13}{|c|}{ Ítems } & \multirow{2}{*}{$\begin{array}{l}\text { Media } \\
\text { aritmética }\end{array}$} & \multirow{2}{*}{$\begin{array}{l}\text { \% respecto a la } \\
\text { muestra }\end{array}$} \\
\hline & & 1 & 2 & 3 & 4 & 5 & 6 & 7 & & 8 & 9 & & 10 & & & \\
\hline Alto & $\begin{array}{l}\text { Domina las } \\
\text { metodologias } \\
\text { activas. }\end{array}$ & 14 & 14 & 13 & 14 & 14 & 14 & 11 & 14 & & & 14 & & 14 & 13 & $92,85 \%$ \\
\hline Medio & $\begin{array}{l}\text { Identifica las } \\
\text { características de } \\
\text { las metodologias } \\
\text { activas. }\end{array}$ & 0 & 0 & 0 & 0 & 0 & 0 & 0 & & 0 & 0 & & 0 & & 0 & $0 \%$ \\
\hline Bajo & $\begin{array}{l}\text { No conoce las } \\
\text { metodologías } \\
\text { activas }\end{array}$ & 0 & 0 & 1 & 0 & 0 & 0 & 3 & & 0 & 0 & & 0 & & 0.4 & $2,85 \%$ \\
\hline
\end{tabular}

Fuente: Elaboración propia

Si bien en esta investigación se reconoce una severa limitación en cuanto al tamaño de la muestra, lo cual impide efectuar generalizaciones probabilísticas, se concluye que se logró capacitar en metodologías activas al cuerpo docente de la Unidad Educativa Reino de Inglaterra. En ausencia de datos similares en investigaciones anteriores la conclusión se sustenta desde la comparación entre los resultados de la comparación entre los indicadores en el pretest y el postest. No obstante a ello, este estudio aporta desde la práctica un diseño de talleres de capacitación que los directivos de la institución pueden disponer para solventar las necesidades de sus docentes en cuanto al tema de metodologías activas.

Finalizando, se comparte la necesidad de seguimiento al personal docente para constatar la incorporación de las metodologías activas a sus planificaciones curriculares y su implementación en el aula para generar aprendizajes significativos. Resultaría interesante también, incrementar la muestra como mínimo a dos unidades particulares y dos fiscales para desarrollar un estudio experimental de mayor trascendencia y generalizar los resultados obtenidos en la presente investigación. 


\section{Conclusiones}

La aplicación de un pretest consistente en un cuestionario de 10 items reveló las carencias acerca del conocimiento que tiene el personal docente de la unidad educativa Reino de Inglaterra, de igual manera este instrumento reveló que son insuficientes las capacitaciones recibidas, lo que genera una necesidad de diseñar un módulo de capacitación sobre metodologías activas.

El módulo de capacitación diseñado contribuyó al conocimiento sobre las metodologías activas en el profesorado de la unidad educativa Reino de Inglaterra. Esto se evidencia en los datos estadísticos obtenidos a partir de un postest de 10 ítems aplicados a los miembros de la muestra.

\section{REFERENCIAS}

Aguilar, E. (2010). El proceso de administración de la capacitación. Ciudad de Juárez, México: A.C.

Campillay, S. y Meléndez, N. (2015). Análisis de impacto de Metodología Activa y Aprendizaje Heurístico en asignaturas de ingeniería. Actualidades Investigativas en educación. 15(2), 1-16. Recuperado de https://doi.org/ 10.15517/AIE.V15I2.18950

Castillo, L. y Puente, D. (30 de julio de 2016). USD 17 millones se invertirán para capacitar a docentes hasta el 2017. El Comercio. Recuperado de https://bit.ly/2XVmRiY

De León, A. (2013). Metodología activa en el proceso de enseñanza aprendizaje y la fundamentación de los estilos de aprendizaje. (Tesis doctoral). Universidad de San Carlos de Guatemala, Guatemala. Recuperado de https://bi t.ly/2IREQke

Díaz, V. (2006). Formación docente, práctica pedagógica y saber pedagógico. Laurus, 12 (extraordinario), 88-103. Recuperado de http://www.redalyc.org/articulo.oa?id=76109906

Escudero, J, (2013). Guia del maestro. España: Publicaciones Universidad Pontificia España

Flotts, P., Manzi, J., Jiménez, D., Abarzúa, A., Cayuman, C. y García, M.J. (2016). Tercer Estudio regional y Comparativo de la Calidad de la Educación. Logros de aprendizaje: Informe de resultados. París, Francia: Acento en la Ce Spa Recuperado de http://unesdoc.unesco.org/images/0024/002435/243532S.pdf

García, A. (2012). Simulación telemática como experiencia de aprendizaje de la lengua inglesa. REDU: Revista de docencia universitaria, 10 (3), 301-323. Recuperado de https://polipapers.upv.es/index.php/REDU/article/vi ew/6025/6090

Hernández R, Fernández C y Baptista P. (2014). Metodología de la Investigación. México: McGraw Hill

Herrera, L. y Lorenzo, O. (2009). Estrategias de aprendizaje en estudiantes universitarios. Un aporte a la construcción del Espacio Europeo de Educación Superior. Educación y Educadores, 12(3), 75-98. Recuperado de http://ww w.redalyc.org/pdf/834/83412235005.pdf

Labrador, J. (2008). Metodologias activas. Valencia: Universidad Politécnica de Valencia.

Lawshe, C. H. (1975). A quantitative approach to content validity. Personnel Psychology, 28, 563-575. Recuperado de https://bit.ly/2IYiMEG

Learreta, B., Montil, M., González, A. y Asensio, A.(2009) Percepción del alumnado ante el uso de metodologías activas de enseñanza como respuesta a las demandas del espacio europeo de educación superior: un estudio de caso. Apunts Educación Física y Deportes. (95): 92-98. Recuperado de: http://www.redalyc.org/articulo.oa?id=551 656931013

Lynn, M. R. (1986). Determination and quantification of content validity. Nursing Research, 35, 382-385. Recuperado de https://bit.ly/2FfbXxs

Ministerio de Educación. (2011). Ley orgánica de educación intercultural. Recuperado de https://bit.ly/2DWohyM

Romero, M. (2010). El aprendizaje experiencial y las nuevas demandas formativas. Revista de Antropología Experimental, 10 (8), 89-102. Recuperado de https://revistaselectronicas.ujaen.es/index.php/rae/article/view $/ 1970 / 1718$ 
Secretaría Nacional de Planificación de la República del Ecuador. (2013). Plan Nacional del Buen Vivir. Recuperado de https://bit.ly/2KmTaEB

Vargas, M. (2009). Métodos de Enseñanza. Miami: Lectorun

CC BY-NC-ND 